\title{
Prevalence of anaemia among HIV-infected patients in Benin City, Nigeria
}

\author{
R. OMOREGIE ${ }^{1 *}$, E.U.OMOKARO ${ }^{1,2}$, O. PALMER ${ }^{1}$, H.O. OGEFERE ${ }^{1}$, A. EGBEOBAUWAYE ${ }^{1}$, \\ J.E. ADEGHE ${ }^{1}$, S.I. OSAKUE ${ }^{1}$ and V. IHEMEJE ${ }^{2}$. \\ ${ }^{1}$ School of Medical Laboratory Sciences, University of Benin Teaching Hospital, \\ P.M.B. 1111, Benin City; Edo State, Nigeria \\ ${ }^{2}$ Department of Haematology and Blood Transfusion Science, University of Benin Teaching Hospital, \\ P.M.B. 1111, Benin City, Edo State, Nigeria
}

\begin{abstract}
The aim of this study was to use the World Health Organization (WHO) definition of anaemia to determine prevalence of anaemia among human immunodeficiency virus (HIV)-infected patients on the highly active antiretroviral therapy (HAART) and those that are HAART naive. Haemoglobin concentration was measured in 457 HIV patients consisting of 217 patients on HAART (86 males and 131 females) and 240 HAART naive patients (106 males and 134 females). According to WHO criteria, anaemia was defined as a haemoglobin concentration below $12 \mathrm{~g} / \mathrm{dl}$ in women and below $13 \mathrm{~g} / \mathrm{dl}$ in men. The anaemic HIV patients were further categorized according to WHO/ACTG anaemia toxicity grades. An overall anaemia prevalence of $60.61 \%$ was observed. The prevalence of anaemia was significantly higher among HAART naive patients $(69.17 \%)$ than in HIV patients on HAART $(51.15 \%)(P<0.001)$. The prevalence of anaemia differ significantly $(P<0.05)$ between males and females of HAART naive patients with males $(76.42 \%)$ having higher prevalence than females (63.43\%). The WHO/ACTG categorization showed the same pattern between HIV patients on HAART and those that were HAART naive. Conclusively, the overall prevalence of anaemia was $60.61 \%$ among HIV patients. HAART naive patients have higher prevalence as well as males in this group. The WHO definition of anaemia is recommended as this will give the true prevalence of anaemia and allow for policy and interventions to address it.
\end{abstract}

Keywords: Anaemia, HAART, HIV, Nigeria

\section{introauction}

Acquired immunodeficiency syndrome (AIDS) is caused by the human immunodeficiency virus (HIV) and is characterized by progressive damage to the body's immune system which results in the development of a number of opportunistic infections and other complications (Okolie et al., 2003). The haematologic complications of HIV-infected patients include anaemia, neutropenia, lymphopenia and thrombocytopenia (Moyle, 2002). Anaemia is the most commonly encountered haematologic abnormalities in HIV patients, occurring with increasing frequency and is a significant predictor of progression to AIDS or death, with more than $70 \%$ of patients developing anaemia and requiring transfusion (Volberding, 2002; Odunukwe et al., 2005).

The treatment of HIV patients with the HAART has generally been taken as the gold standard in the management of HIV patients (Odunukwe et al., 2005). HAART have been reported to improve haematocrit and haemoglobin values as well as result in significantly reduction in morbidity and mortality of HIV patients (Gea - Banacloche \& Lane, 1999; Odunukwe et al., 2005). The use of HAART has also been reported to cause mild to moderate anaemia (Mildvan, 2003) and some authors report no improvement in haematocrit values of HAART treated HIV patients when compared with their HAART naïve counterparts (Omoregie et al., 2008).

The available literature on the prevalence of anaemia among HAART treated and HAART naive HIV patients show conflicting values as different definitions of anaemia based on the haemoglobin concentration were used (Nadler et al., 2003; Curkendall et al., 2007). Against this background, this study aimed to use the World Health Organisation (WHO) definition of anaemia to determine the prevalence of anaemia among HAART naive and HAART-treated HIV patients in Benin City, Nigeria.

\section{Materials and Methods}

\section{Study area}

The study was carried out in the University of Benin Teaching Hospital (UBTH) Benin City, Nigeria. The hospital as a tertiary teaching hospital has a referral status and is located in the South-South geopolitical zone of Nigeria. It serves about $6-10$ states within and outside

\footnotetext{
*Correspondence: Richard Omoregie; E-mail:: richyomos@yahoo.com.
} 
the zone. It is a centre for Institute of Human Virology, Nigeria (IHVN) and President's Emergency Plan For AIDS Relief (PEPFAR) HIV/AIDS interventions in the zone. HIV/ AIDS interventions include free diagnosis, treatment and monitoring. The centre diagnoses new cases and monitors those on therapy. Some of these patients were used for this study.

\section{Study population}

A total 457 HIV patients attending HIV clinics in the University of Benin Teaching Hospital (UBTH), Benin City, Nigeria, were recruited for this study. The patients consisted of 217 (86 males and 131 females) HIV patients on HAART for 3 - 6 months and 240 (106 males and 134 females) HAART naïve HIV patients. The age range of the patients was $19-61$ years with a mean of $30.57 \pm 7.81$ years. Five millilitres of blood was collected from each patient and dispensed into ethylene diamine tetra-acetic acid (EDTA) containers and mixed. The HAART regimen for HIV patients on HAART consist of zidovudine, stavudine and nevirapine. Patients, who had received blood transfusion within the last 3 months, were on erythropoietin therapy and having any opportunistic infection were excluded from the study.

\section{Determination of haemoglobin concentration and definition of anaemia}

The haemoglobin concentration of the collected samples was determined using an autoanalyserSysmex KX-21 (Sysmex Corporation, Kobe, Japan). Anaemia was defined according to the WHO criteria (Izaks et al., 1999). For males anaemia was defined as haemoglobin concentration $(\mathrm{Hb})$ less than $13 \mathrm{~g} / \mathrm{dl}$, while for females, the value is less than $12 \mathrm{~g} / \mathrm{dl}$. The HIV patients that were anaemic were further classified according to WHO/ACTG anaemia toxicity grades (Moyle, 2002). The grades are as follow; grade $1(\mathrm{Hb}$ of $9.5-10.5 \mathrm{~g} / \mathrm{dl})$, grade 2 $(\mathrm{Hb}$ of $8-9.4 \mathrm{~g} / \mathrm{dl})$, grade $3(\mathrm{Hb}$ of $6.5-7.9 \mathrm{~g} / \mathrm{dl})$ and grade $4(\mathrm{Hb}$ of $<6.5 \mathrm{~g} / \mathrm{dl})$.

\section{Statistical analysis}

The Chi $\left(X^{2}\right)$ Square test was used to analyze the data obtained. The data were arranged in a $2 \times 2$ contingency table before manual analysis. For example, the HIV patients were grouped as HAART naïve and on HAART, and further grouped into those with and without anaemia.

\section{Ethical considerations}

The Ethical Committee of the University of Benin Teaching Hospital Benin City, Nigeria, approved the protocol for this study. Verbal informed consent was obtained from every patient used in this study.

\section{Results}

An overall prevalence of $60.61 \%$ (277 out of 457 patients) of anaemia was observed in this study. HIV patients on HAART had a significantly lower prevalence of anaemia compared with their HAART naive counterparts $(51.15 \%$ Vs $69.17 \%$, $P<0.001)$. Generally, there was no significant difference in the prevalence of anaemia between both genders $($ male $=61.98 \%$; female $=59.62 \%)$. However, male HAART naive HIV patients had significantly higher prevalence of anaemia than females in the same group $(76.42 \%$ Vs $63.43 \%$, $P<0.05$ ) (Table 1).

Table 1: Prevalence of anaemia among HIV patients

\begin{tabular}{|c|c|c|c|c|c|c|}
\hline \multirow[t]{2}{*}{ HIV patients } & \multicolumn{2}{|l|}{ Male } & \multicolumn{2}{|l|}{ Female } & \multicolumn{2}{|l|}{ Total } \\
\hline & No. tested & $\begin{array}{l}\text { No. with } \\
\text { anaemia (\%) }\end{array}$ & No. tested & $\begin{array}{l}\text { No. with } \\
\text { anaemia(\%) }\end{array}$ & No. tested & $\begin{array}{l}\text { No. with } \\
\text { anaemia (\%) }\end{array}$ \\
\hline On HAART & 86 & $38(44.19)$ & 131 & 73 (55.73) & 217 & $111(51.15)$ \\
\hline HAART naïve & 106 & $81(76.42)$ & 134 & $85(63.43)$ & 240 & $166(69.17)$ \\
\hline Total & 192 & 119 (61.98) & 265 & 158 (59.62) & 457 & 277 (60.61) \\
\hline
\end{tabular}


A total of $182(65.70 \%)$ out of the 277 anaemic HIV patients fell within the $\mathrm{WHO} / \mathrm{ACTG}$ anaemia grades. The prevalence of anaemia within the grades did not differ significantly between HIV patients on HAART and those that were HAART naive $(P>0.05)$, (table 2$)$. Generally, the prevalence of anaemia dropped between grade 1 and 4 of the WHO/AGTG grades (Table 2). anaemia definition may be responsible for the difference in the results. It is important to note that the lower prevalence of anaemia among HIV patients receiving HAART, may indicate the effectiveness of the HAART therapy in reducing viral load and improving haemotocrit values and it has been reported that HAART increase haemoglobin concentration and

Table 2: Prevalence of anaemia among HIV patients according to WHO/ACTG toxicity grades for anaemia

\begin{tabular}{lll}
\hline WHO/ACTG Anaemia grade & HIV patients on HAART & HAART naive HIV \\
Grade $1(\mathrm{Hb}=9.5-10.5 \mathrm{~g} / \mathrm{dl})$ & $37(51.39 \%)$ & $48(43.64 \%)$ \\
Grade $2(\mathrm{Hb}=8-9.4 \mathrm{~g} / \mathrm{dl})$ & $26(36.11 \%)$ & $45(40.91 \%)$ \\
Grade $3(\mathrm{Hb}=6.5-7.9 \mathrm{~g} / \mathrm{dl})$ & $8(11.11 \%)$ & $16(14.55 \%)$ \\
Grade $4(\mathrm{Hb}=<6.5 \mathrm{~g} / \mathrm{dl})$ & $1(1.39 \%)$ & $1(0.91 \%)$ \\
\hline
\end{tabular}

\section{Discussion}

Anaemia is the most commonly encountered haematologic abnormality in HIV patients (Volberding, 2002). HAART which has been taken as the gold standard in the management of HIV patients was reported to improve haematocrit (Odunukwe et al., 2005). However, Omoregie et al. (2008) observed that HAART did not improve haematocrit of HIV patients and Mildvan (2003) reported that HIV patients on HAART still develop mild to moderate anaemia.

In this study an overall prevalence of anaemia of $60.61 \%$ was higher than that reported by Nadler et al. (2003). This difference may be due to the definition of anaemia as Nadler et al. (2003) anaemia was defined as $\mathrm{Hb} \leq 12.5 \mathrm{~g} /$ $\mathrm{dl}$ for both males and females. It is important that a unified definition of anaemia, such as the WHO definition, be used. Also, in Nadler et al. (2003) report, not all patients on HAART had zidovudine (AZT) in their regimen as was the case in this study. This is important as AZT has been reported by several authors to cause anaemia by inhibition of haemoglobin synthesis and toxicity to bone marrow cells, particularly, erythroid lines (Moyle, 2002; Omoregie et al., 2008).

HAART naïve HIV patients had a significantly higher prevalence of anaemia $(69.17 \%)$ than HIV patients on HAART. This finding is not in agreement with that of Nadler et al. (2003), in which no significant difference in the prevalence of anaemia was observed between HIV patients on HAART and their HAART naive counter parts. Again the difference in decreases the prevalence of anaemia (Belperio \& Rhew, 2004; Odunukwe et al., 2005). A number of mechanisms have been suggested to explain the prevalence of anaemia among HIV patient on HAART. They include the presence of antibodies to HAART agents (Omoregie et al., 2008), the presence of AZT among the HAART regimen and CD4 counts (Moyle, 2002; Nadler et al., 2003; Curkendall et al., 2007). However, reports concerning the relationship between CD4 count and anaemia are conflicting. Moyle (2002) reports that anaemia is independent of CD4 count and viral load. Nadler et al. (2003) and Curkendall et al. (2007) reports that CD4 count is a predictor of anaemia. However, the values for CD4 count differ. While Nadler et al. (2003) report that CD4 count $<50$ cells $/ \mu \mathrm{L}$ is a significant predictor of anaemia, a CD4 count of $<200$ cells $/ \mu \mathrm{L}$ was the value associated with anaemia in Curkendall et al. (2007) report. Further studies are needed to resolve the effect of $\mathrm{CD} 4$ count on the prevalence of anaemia.

Female gender has been reported as a risk factor for anaemia among HIV patients (Nadler et al., 2003). In this study, overall, there was no significant difference in the prevalence of anaemia between male and females. The same picture was observed among HIV patients receiving HAART. However, among HAART naive HIV patients, males had significantly higher prevalence of anaemia than their female counterparts. The findings in this study differ from that of Nadler et al. (2003) most probably due to the difference in the definition of anaemia. 
Two-thirds of the anaemic HIV patients fell within the WHO/ACTG anaemia grades. Studies indicates that individuals with haemoglobin values below the normal range but not in toxicity grade 1, still show symptoms of anaemia (Moyle, 2002). There was no significant difference in the prevalence of anaemia within the WHO/ACTG toxicity grades between HIV patients receiving HAART and those that are HAART naïve. This may indicate a similar anaemia aetiology that will require further investigation.

In conclusion, HAART naive HIV patents have significantly higher prevalence of anaemia than those receiving HAART. Gender difference in the prevalence of anaemia was observed only among HAART naive HIV patients with significant higher prevalence of anaemia observed among males. A unified definition of anaemia, such as the WHO definition, is advocated as this will give the true prevalence of anaemia and allow for policy and interventions to address it.

\section{Acknowledgements}

We acknowledge with thanks the Management of University of Benin Teaching Hospital for permission to carry out this study.

Received 1 June 2008

Revised 24 October 2008

Accepted 25 November 2008

\section{References}

Belperio, P.S. \& Rhew, D.C. (2004) Prevalence and outcomes of anaemia in individuals with human immunodeficiency virus; a systematic review of the literature. American Journal of Medicine 16, 27 - 43.

Curkendall, S.M., Richardson, J.T., Emons, M.F., Fisher, A.E. \& Everhard, F. (2007) Incidence of anaemia among HIVinfected patients treated with highly active antiretroviral therapy. HIV Medicine 8, 483 - 490.
Gea - Banacloche, J.C. \& Lane, H.C. (1999). Immune reconstitution in $\mathrm{HIV}-1$ infections. AIDS 13, $525-538$.

Izaks, G.J., Westendorp, R.G.J. \& Knooks, D.L. (1999) The definition of anaemia in older persons. Journal of the American Medical Association. 281, 1714 - 1717.

Mildvan, D. (2003). Implications of anaemia in human immunodeficiency virus, cancer and hepatitis $C$ virus. Clinical and Infectious Diseases 37, 293 - 296.

Moyle, G. (2002) Anaemia in person with HIV infection: prognostic marker and contributor to morbidity. AIDS Review 4, $13-20$.

Nadler, J.P., Wills T.S., Somboonwit, C., Vincent A., Lietz, G., Marino, K., Naik, E., Powers, S., Khan, N., Almyroudis, N \& Laatz, B. (2003) Anaemia prevalence among HIV patients: antiretroviral therapy and other risk factors. Antiviral Therapy 8 (suppl 1).

Odunukwe, N., Idigbe, O., Kanki, P., Adewole, T., Onwujekwe, D., Audu, R. \& Onyewuche, J. (2005) Haematological and biochemical response to treatment of HIV-1 infection with a combination of nevirapine + stavudine + lamivudine in Lagos, Nigeria. Turkish Journal of Haematology 22, 125 - 131.

Okolie, M.N., Eghafona, N.O. \& Omoregie, R. (2003) Anti-Human immunodeficiency virus agents. Journal of Medical Laboratory Science 12, 1-14.

Omoregie, R., Egbeobauwaye, A., Ogefere, H., Omokaro E.U. \& Ekeh, C.C. (2008) Prevalence of antibodies to HAART agents among HIV patients in Benin City, Nigeria. African Journal of Biomedical Research. 11, 33-37.

Volberding, P. (2002) The impact of anaemia on quality of life in human immunodeficiency virus infected patients. Journal of Infectious Disease 185, 110-114. 